\title{
Effect of low-dose ketamine versus fentanyl on attenuating the haemodynamic response to laryngoscopy and endotracheal intubation in patients undergoing general anaesthesia: a prospective, double-blinded, randomised controlled trial
}

\author{
Angela Ongewe, Vitalis Mung'ayi, Rajpreet Bal
}

Department of Anaesthesia, Aga Khan University, East Africa.

\begin{abstract}
Author details:
Email: angela.ongewe@aku.edu, Phone:+254 20366 2158; Email: vitalis.mung'ayi@aku.edu, Phone: +254 20 366 2175; Email: rajpreet.bal@aku.edu, Phone: + 254203662158.
\end{abstract}

\begin{abstract}
Background: The use of drugs to attenuate the haemodynamic response to laryngoscopy and endotracheal intubation is the standard of care during elective surgery. Current evidence is conflicting concerning the best agent and optimal dose for this purpose. In the majority of cases, fentanyl is widely utilized to attenuate haemodynamic responses. Ketamine, an established available drug, has been scarcely studied in this regard at low doses and against varying doses of other common agents.

Objective: To compare the overall occurrence of hypertension and tachycardia immediately pre-intubation (post-induction) until 10 minutes post intubation between the study group receiving fentanyl at $1.0 \mu \mathrm{g} / \mathrm{kg}$ and the other receiving ketamine at $0.5 \mathrm{mg} / \mathrm{kg}$, to compare the occurrence of post-induction hypotension and the occurrence of neuropsychiatric phenomena during emergence between the two groups.

Methods: One hundred and eight ASA I and II patients aged 18-65 years scheduled for elective surgery under general anaesthesia were randomized into two groups: Control group: received fentanyl $1.0 \mu \mathrm{g} / \mathrm{kg}$ intravenously. Intervention group: received ketamine $0.5 \mathrm{mg} / \mathrm{kg}$ intravenously. General anaesthesia was standardized in both groups. The patients and physicians administering anaesthesia were blinded to the study. Haemodynamic responses were evaluated by determining heart rate and blood pressure immediately before laryngoscopy and at 2.5, 5, 7.5 and 10 minutes. Neuropsychiatric phenomena were assessed upon recovery from anaesthesia. Results: One hundred and eight ASA I and II patients scheduled to undergo elective surgery were included in this study, 54 participants $(50 \%)$ in the fentanyl arm and $54(50 \%)$ in the ketamine arm. Baseline demographic characteristics were similar between the groups. There were more hypertensive episodes in the ketamine arm (11\%) compared to the fentanyl arm (1.85\%), but not achieving statistical significance: Fisher's exact test, $\mathrm{p}=0.06$. There was no significant difference in the number of episodes of tachycardia between the Ketamine group 7/54 (13\%) and the fentanyl group, 6/54 (11\%); $2=0.05, \mathrm{p}=0.82$. Hypotensive episodes were more common in those who received Fentanyl, 41/54 (76\%), compared to ketamine recipients, $21 / 54$ (39\%), X2=16.9, $<<0.001$. The use of Ketamine was associated with less episodes of hypotension, adjusted odds ratio $=0.18$ ( $95 \%$ confidence interval $0.07,0.45)$. Conclusion: We conclude, based upon findings in this study group, that there is no difference in the occurrence of hypertension with the use ketamine at $0.5 \mathrm{mg} / \mathrm{kg}$ in combination with Propofol at $2.0 \mathrm{mg} / \mathrm{kg}$. In this regard, ketamine provides a viable alternative to fentanyl at $1.0 \mu \mathrm{g} / \mathrm{kg}$ for attenuating the pressor response to laryngoscopy and endotracheal intubation. Additionally, our results suggest that ketamine may protect against post-induction (pre-laryngoscopy) hypotension.
\end{abstract}

Keywords: Low-dose ketamine, fentanyl, laryngoscopy, endotracheal, intubation, double-blinded, randomised controlled trial. DOI: https://dx.doi.org/10.4314/ahs.v19i3.51

Cite as: Ongewe A, Mung'ayi V, Bal R. Effect of low-dose ketamine versus fentanyl on attenuating the haemodynamic response to laryngoscopy and endotracheal intubation in patients undergoing general anaesthesia: a prospective, double-blinded, randomised controlled trial. Afri Health Sci. 2019;19(3): 2752-2763. bttps://dx.doi.org/10.4314/abs.v19i3.51
Correspondence author:
Vitalis Mung'ayi,
Department of Anaesthesia, Aga Khan
University, East Africa
Phone: +254 20366 2175;
Email: vitalis.mung'ayi@aku.edu

\section{Introduction}

General anaesthesia with direct laryngoscopy and endotracheal intubation (LTI) is frequently used in surgical patients. Even with a trend towards increased use of regional and local anaesthetic techniques (with or without sedation), general anaesthesia with LTI remains the choice for several forms of surgery e.g. maxillofacial sur-

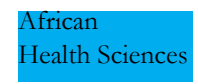

2752
(C) 2019 Ongewe et al. Licensee African Health Sciences. This is an Open Access article distributed under the terms of the Creative commons Attribution License (https://creativecommons.org/licenses/BY/4.0), which permits unrestricted use, distribution, and reproduction in any medium, provided the original work is properly cited.
African Health Sciences Vol 19 Issue 3, September, 2019 
gery, cardiothoracic surgery, laparoscopic abdominal surgery and procedures requiring rapid sequence induction1. Laryngoscopy and endotracheal intubation is one of the most haemodynamically stimulating points during the course of the general anaesthesia. The mechanisms proposed include a somato-visceral reflex, stimulation of proprioceptors at the base of the tongue with catecholamine release and vagal inhibition of the heart ${ }^{2}$. This provokes a haemodynamic response characterized by an increase in heart rate (up to $83 \%$ ) and blood pressure (up to $80 \%$ ) These haemodynamic changes typically start within $5 \mathrm{sec}-$ onds of laryngoscopy and peak $1 \mathrm{~min}$ after initiation of intubation and persist for 5-10 minutes $^{4-6}$. This may lead to myocardial ischemia, ventricular arrhythmia, left ventricular failure and cerebral haemorrhage ${ }^{7}$.

Various factors affect the magnitude of this response. A variety of anaesthetic techniques and drugs have been studied and are available to control the hemodynamic response to laryngoscopy and intubation ${ }^{7}$. Induction drugs may attenuate adverse hemodynamic responses to laryngoscopy and endotracheal intubation ${ }^{7}$. Well studied agents/modalities include opioids, local anaesthetics, beta blockers, calcium channel blockers, benzodiazepines, barbiturates, alpha adrenergic antagonists, vasodilators, alpha 2 agonists, angiotensin converting enzyme inhibitors, lornoxicam, inhalational anaesthetics, pregabalin and nerve blocks ${ }^{3,8-14}$. In a 2013 Cochrane review of $72 \mathrm{RCTs}^{16}$, all studies reporting arrhythmias were analysed and 49 trials enrolling lower risk patients revealed a significant difference observed between the placebo and treatment groups (OR 0.20, 95\% CI 0.14 to $0.28, \mathrm{P}<0.00001$, I2 $=44 \%$ ). This review suggests that even low risk (ASA I/II) patients are susceptible to cardiac arrhythmia and ECG evidence of myocardial ischaemia as a consequence of haemodynamic changes related to tracheal intubation. Of these low risk patients, $13 \%$ had arrhythmias and 1.4 $\%$ had myocardial ischaemia when a placebo was given prior to laryngoscopy and intubation. Pre-induction use of pharmacological agents reduced this risk to $4.5 \%$ for arrhythmias and nil for myocardial ischaemia ${ }^{16}$.

These drugs and their various combinations have been tried in the past and studies have highlighted their use in varying doses for the suppression of this stress response but with significant occurrence of adverse effects, especially with use of higher doses of opioids ${ }^{9}$.
Fentanyl is a synthetic opioid analgesic with a potency of approximately $75-125$ times that of morphine. It is the most commonly used agent in Aga Khan University Hospital, Nairobi, for attenuating this pressor response.

Ketamine, a phencyclidine derivative, is an N-Methyl D-Aspartate receptor antagonist that also acts at numerous other sites. It inhibits opioid mu receptors while stimulating delta and kappa receptors. Ketamine is in wide use as an anaesthetic, sedative and analgesic agent. Intravenous induction doses for anaesthesia range from $1-2 \mathrm{mg} / \mathrm{kg}$ (with an intramuscular induction dose of $5-10 \mathrm{mg} / \mathrm{kg}$.) Ketamine is widely available yet has scarcely been soundly studied as an agent for the attenuation of haemodynamic response to $\mathrm{LTI}^{20}$. Indeed, it is known to cause hypertension and tachycardia at anaesthetic doses. This cardio-stimulatory effect is however minimal to nil at sub-anaesthetic/analgesic doses ${ }^{15}$.

This study was designed to compare the overall occurrence of hypertension in response to laryngoscopy and endotracheal intubation between low-dose ketamine versus fentanyl in patients undergoing general anaesthesia. Considering the enormous effect of anaesthetic drugs on the patients' haemodynamics during and after intubation, this study intends to explore a possible alternative regimen to reduce this morbidity. Our study question is: does low-dose ketamine result in lower occurrence of post laryngoscopy and intubation tachycardia and hypertension compared to fentanyl?

We hypothesized that there is no difference in the occurrence of post-laryngoscopy and intubation hypertension and tachycardia in patients who receive pre-laryngoscopy and intubation low dose ketamine versus fentanyl. Our primary objective was to compare the effect of low-dose ketamine versus fentanyl on the occurrence of hypertension in patients undergoing general anaesthesia. Our secondary objectives were to compare the occurrence of tachycardia between the two study groups, compare the occurrence of pre-laryngoscopy and intubation hypotension between the two study groups and to compare the occurrence of neuropsychiatric phenomena upon recovery from anaesthesia.

\section{Methods}

The study was performed following approval from the re- 
search scientific and ethical committees of the Aga Khan University, East Africa. This was a prospective, double blinded, and randomized controlled trial. Patients were recruited from the outpatient pre-anaesthesia clinics as well as the day care and in-patient wards. The target population included patients admitted for elective and urgent surgery under general anaesthesia with laryngoscopy and endotracheal intubation at the Aga Khan University Hospital, Nairobi. The sample population included American Society of Anesthesiologists (ASA) physical status (PS) I and II patients going to theatre for elective surgery between November 2015 and April 2016.

All ASA I and II patients 18-65 years scheduled for elective surgery aged between 18 and 65 years were included in the study. Reasons for exclusion from the study were:

1. History of allergy or contraindications to any of the study drugs.

2. Patients scheduled for emergency surgery.

3. Age below 18 years and above 65 years.

4. Hypertension - a known/previously diagnosed hypertensive as per the American Heart Association guidelines.

5. Pregnancy.

6. Dementia/psychiatric illness.

7. Known ischaemic heart disease.

8. Known raised intracranial pressure or intracranial pathology where a rise in intracranial pressure would pose a risk or patients scheduled for neurosurgical procedures. 9. History, indicator (Mallampati score) of or proven difficult ventilation/laryngoscopy/intubation.

10. Intubation time greater than 30 seconds/Repeat attempts at intubation.

11. Patients who did not agree to the clinical trial.

Previous studies show incidence of tachycardia and hypertension of $55 \%$ and $40 \%$ respectively with fentanyl (versus $85 \%$ and $80 \%$ respectively with controls). ${ }^{3}$ This represents a $30 \%$ decrease in tachycardia and hypertension than control. This study sought to identify a further $30 \%$ decrease in these haemodynamic parameters with use of low dose ketamine (compared to Fentanyl). Using this information and assuming 5\% significance level and power of $80 \%$, a sample size of 80 patients was found sufficient to demonstrate a $30 \%$ difference in the incidence of hypertension and of tachycardia between pa- tients undergoing general anaesthesia with LTI given low dose Ketamine versus Fentanyl. The study was powered at $80 \%$. Type 1 error was set at 0.05 . Accounting for a $10 \%$ drop-out gave us a total sample size of 88 with 44 patients in each study arm.

Study participants were recruited after screening to determine eligibility and obtaining informed consent for the study. Participants were screened during the pre-operative anaesthesia assessment session in the anaesthesia clinic, in-patient wards and day care unit. Consecutive sampling was employed such that every available subject eligible for selection was recruited. All potential participants received a verbal and written explanation on the purpose and procedure of the study from the principal investigator and written informed consent sought. The patients who gave written informed consent were enrolled into the study and then randomized.

The participants were randomly allocated to either arm using simple randomization. A random allocation sequence was generated by a statistician using a computer algorithm and consenting participants were allocated to either arm based on the random allocation sequence. The principal investigator was not engaged in the generation of the random allocation sequence. The statistician had envelopes that were serialized to correspond to the aforementioned random allocation sequence. Once a patient gave informed consent, the clinician screening the patient attached the envelope to the anaesthesia chart/data collection tool/consent form. Once the participant arrived in the pre-operative receiving area of the main theatres, a research assistant not involved in any other aspect of the study received these envelopes containing the allocation number that he used to know what drug to give according to the allocation chart available to him only. He then proceeded to prepare the appropriate drug up to a volume of $10 \mathrm{~mL}$. Identical $10 \mathrm{~mL}$ syringes labelled "Study Drug" were used. The drug was then presented to the anaesthesiologist in the operating theatre. Both Fentanyl and Ketamine are colourless/clear liquids and so the presentation was identical. In this way, the participant, principal investigator and the anaesthesiologist in the operating theatre remained blinded to the group allocation. Flow diagram of patient distribution is shown in figure 1 . 


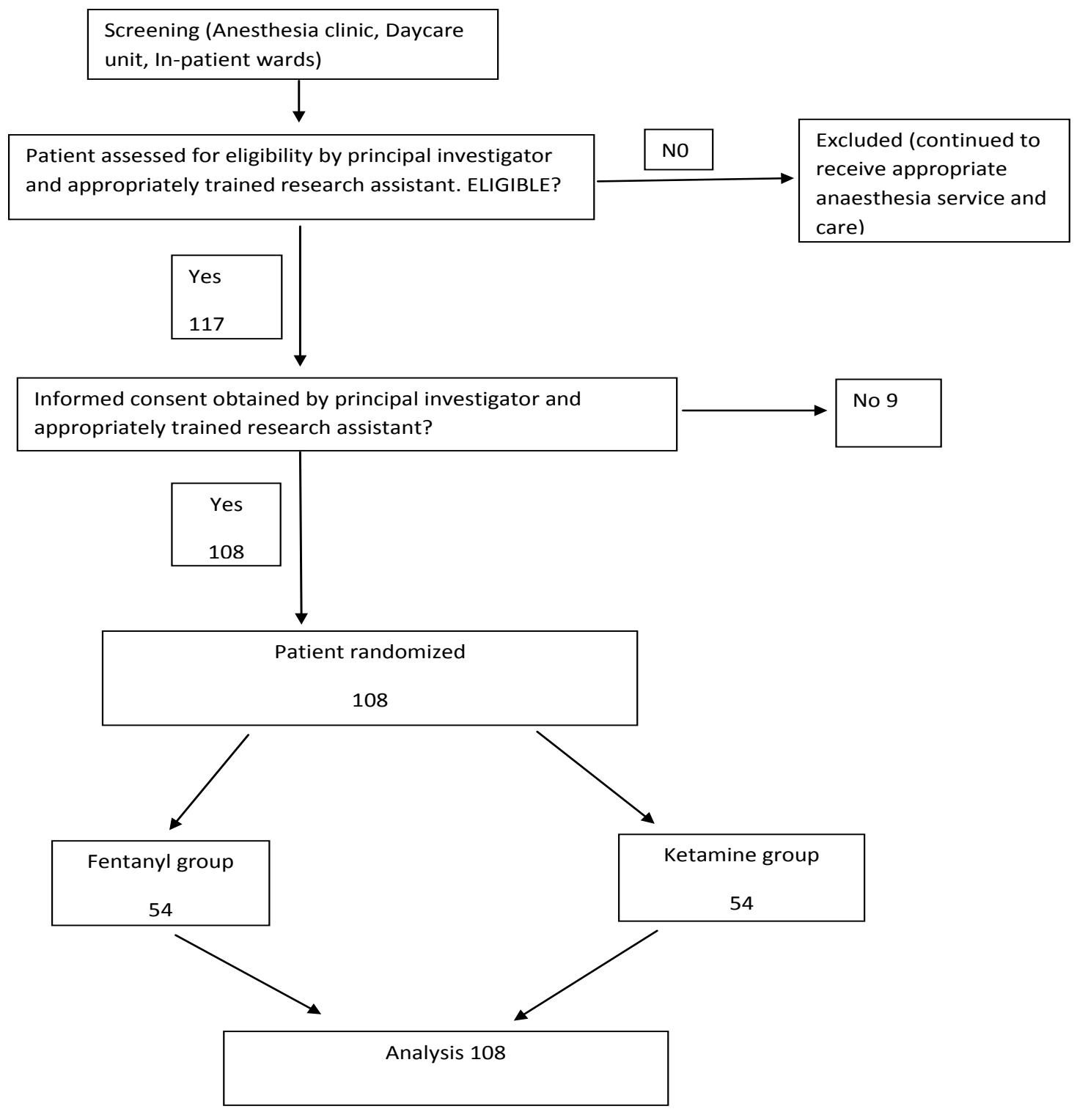

Figure 1. Flow diagram of patient distribution

On arrival in the operating theatre, standard monitoring was applied with automated non-invasive blood pressure measurement, electrocardiography and pulse oximetry, with the objective of obtaining the baseline cardiovascular parameters. Baseline blood pressure was obtained by taking three readings and calculating an average of the three readings.

Intravenous access was obtained and $10 \mathrm{~mL} / \mathrm{kg}$ of Normal Saline or Ringer's Lactate administered over the induction period.
At least 3 minutes of pre-oxygenation was performed. Fentanyl group participants received $1.0 \mu \mathrm{g} / \mathrm{kg}$ fentan$\mathrm{yl}$ and $2.0 \mathrm{mg} / \mathrm{kg}$ propofol as intravenous bolus doses 5 minutes prior to laryngoscopy. Ketamine group participants received $0.5 \mathrm{mg} / \mathrm{kg}$ ketamine and $2.0 \mathrm{mg} / \mathrm{kg}$ propofol as intravenous bolus doses 5 minutes prior to laryngoscopy. $0.2 \mathrm{mg} / \mathrm{kg}$ Cisatracurium was administered as an intravenous bolus dose within 1 minute of administering Fentanyl/Ketamine and Propofol.

Ventilation with 1\% Isoflurane in oxygen via a Mapleson A circuit was initiated followed by laryngoscopy with an 
appropriately sized Macintosh laryngoscope and endotracheal intubation with an appropriately sized cuffed endotracheal tube. This was to commence 3 minutes after the administration of muscle relaxant.

Haemodynamic and other monitoring parameters were observed continuously and recorded at 1 minute post induction (on administration of study drug and Propofol) and then at intervals of 2.5 minutes each for the first 10 minutes.

Upon reversal of anaesthesia (recovery in the post-anaesthesia care unit - PACU) the patient was observed for any neuropsychiatric manifestations such as hallucinations and confusion not attributed to the usual post-general anaesthesia emergence. This information was recorded in the data collection tool.

Any episode of hypertension persisting more than 20 minutes was to be managed by deepening the plane of anaesthesia with Isoflurane or if refractory, with $10 \mathrm{mg}$ boluses of intravenous labetalol titrated to effect. Any incidence of persistent hypotension was to be managed either $3 \mathrm{mg}$ intravenous boluses of Ephedrine or $50 \mathrm{mcg}$ intravenous boluses of Phenylephrine. Neuropsychiatric phenomena were to be identified upon recovery as either hallucinations or confusion not attributed to the realm of immediate recovery after general anaesthesia. These were to be managed with $1 \mathrm{mg}$ intravenous boluses of Midazolam. Pre-emptive use of Midazolam (after the end of the study) could be administered at the discretion of the anaesthesiologist. Any complications that occurred were to be noted by the anaesthesiologist.

Intraoperative data was collected by the anaesthesiologist administering anaesthesia using the data collection form. The principal investigator verified all raw data entered was complete. Data analysis was undertaken using the SPSS version 15. General baseline demographic characteristics (age, sex, ASA status) between the two groups were expressed as percentages. Baseline characteristics between the two groups were summarised as proportions for categorical variables and as means ( \pm standard deviation) or medians (interquartile ranges) for normally distributed and non-normally distributed continuous variables respectively.

Characteristics of patients who received ketamine and fentanyl were compared using chi-squared test or Fisher's exact test for proportions as appropriate. Fisher's exact test was used when any cell value in the two by two ta- ble was $\leq 5$. Student t-test was used to compare means for normally distributed continuous variables between patients who received Fentanyl and those who received Ketamine. Non-parametric test, Wilcoxon rank-sum test, was used to compare medians between the two groups for non-normally distributed continuous variables.

The primary outcome was increase of SBP of more than $20 \%$ from the baseline (considered clinically significant).

Proportions in the ketamine and fentanyl arms with primary outcome were compared using the Fisher's exact test. Similar comparison of proportions was used for secondary outcomes which included the following: the occurrence of tachycardia (heart rate increase more than $20 \%$ from baseline) between the two study groups; occurrence of post-induction, pre-laryngoscopy hypotension (decrease in MAP of more than $20 \%$ ) between the two study groups; and incidence of neuropsychiatric phenomena upon recovery from anesthesia. Chi-square test was used for comparison of proportions where appropriate (when all cell values in the two by two table were $>5$ ). The association between the drugs (Ketamine or Fentanyl) and hypertension, hypotension and tachycardia was assessed by calculation of odds ratios (and 95\% confidence intervals). Multivariable logistic regression was done, and odds ratios calculated, to test for independent association between the drugs and the outcome of interest (hypertension, hypotension and tachycardia). Variables adjusted for in the multivariable model were age, ASA status and sex.

Graphical representations showing changes in SBP, MAP, DBP and heart rates over times between Ketamine and Fentanyl groups were also done. Time to event analyses were done and Kaplan Meier survival curves drawn to compare time to development of hypertension or hypotension between the two groups. Logrank test was used for statistical comparison of time

\section{Results}

One hundred and eight ASA I and II patients scheduled to undergo elective surgery were included in this study. There were 54 participants $(50 \%)$ in the Fentanyl arm and $54(50 \%)$ in the Ketamine arm. Fifty four percent (58) were female, $88(82 \%)$ had ASA I status, none had neuropsychiatric manifestations, median age was 37 years (interquartile range, 29-46), and the mean body weight was $68.2( \pm 12.3)$ kilogram. 
Patients enrolled to Ketamine and Fentanyl groups were similar in sex, ASA status, weight and had similar blood pressure at baseline as shown in table 1 below. Patients randomised to the Ketamine group were generally older than those randomised to the Fentanyl arm but this did not achieve statistical significance.

Table 1: Comparison of baseline characteristics between fentanyl and ketamine groups

\begin{tabular}{|l|l|l|l|}
\hline \multirow{2}{*}{ Baseline patients characteristics } & Fentanyl group & Ketamine group & \multirow{2}{*}{ p value } \\
\cline { 2 - 3 } & $\mathbf{n}(\%)$ & $\mathbf{n}(\mathbf{\%})$ & 0.85 \\
\hline Female Sex & $28(52)$ & $30(56)$ & \\
\hline ASA I & $44(81)$ & $44(81)$ & 0.93 \\
\hline ASA II & $10(19)$ & $10(18)$ & p value \\
\hline & Mean( \pm SD) & Mean( \pm SD) & 0.053 \\
\hline Age (years) & $34.4(9.5)$ & $38.2(10.8)$ & 0.871 \\
\hline Weight (kg) & $68.6(11.0)$ & $68.2(12.3)$ & 0.255 \\
\hline Systolic Blood Pressure (mmHg) & $124.4(16.6)$ & $121.0(14.3)$ & 0.967 \\
\hline Diastolic Blood Pressure (mmHg) & $76.5(9.2)$ & $76.5(9.6)$ & 0.588 \\
\hline Mean Blood Pressure (mmHg) & $92.5(11.1)$ & $91.4(10.3)$ & 0.74 \\
\hline Heart Rate (bpm) & $76.6(10.5)$ & $75.9(10.6)$ & \\
\hline
\end{tabular}

Seven participants developed hypertension ( $>20 \%$ in- of follow up; 6/54 in the Ketamine arm (11.11\%) and crease in baseline) after induction during the duration $1 / 54$ was in the Fentanyl arm (1.85\%) as shown in table 2.

Table 2: Proportion of hypertension between fentanyl and ketamine groups

\begin{tabular}{|l|l|l|l|}
\hline & \multicolumn{1}{|c|}{$\begin{array}{c}\text { Fentanyl } \\
\mathbf{n}(\%)\end{array}$} & \multicolumn{1}{|c|}{$\begin{array}{c}\text { Ketamine } \\
\mathbf{n ( \% )}\end{array}$} & $\begin{array}{c}\text { Total } \\
\mathbf{n ( \% )}\end{array}$ \\
\hline Hypertension post-baseline Absent & $52(51)$ & $49(49)$ & 101 \\
\hline Hypertension post-baseline Present & $1(14)$ & $6(86)$ & 7 \\
\hline Total & Fisher's exact test, $\mathbf{p}=\mathbf{0 . 0 6}$ & 108 \\
\hline
\end{tabular}

There were more hypertensive episodes in the Ketamine arm compared to the Fentanyl arm, but not achieving statistical significance: Fisher's exact test, $\mathrm{p}=0.06$.

The cases with hypertension were mainly due to increase in mean arterial pressure $(n=5)$ and only 1 case each was seen for hypertension due to increase in systolic or diastolic blood pressures. The patient who developed systol- ic hypertension did so at 7.5 minutes but this was normal at 10 minutes and the one who developed diastolic hypertension did so from 1 minute of follow up but had normalised by 10 minutes. At 1 minute, two participants had hypertension diagnosed from increase in mean blood pressure but these resolved by 7 minutes when 3 others had hypertension and two persisted until 10 minutes. 
While accounting for confounders, the association of use of Ketamine with hypertension after adjusting for sex, age and ASA status produced an adjusted odds ratio = $6.36(95 \%$ confidence interval $0.71,57.4)$. This was not statistically significant.
Mean blood pressures were higher post-baseline in the Ketamine group compared to the Fentanyl group as summarised in table 3. This study was however not powered to detect this difference and indeed, this was not deemed clinically significant given that the overall occurrence of hypertension was not statistically significantly higher with the Ketamine than the Fentanyl group.

Table 3: Comparison of blood pressure between fentanyl and ketamine groups

\begin{tabular}{|c|c|c|c|}
\hline \multirow{2}{*}{$\begin{array}{l}\text { Time }(\min ) \text { post- } \\
\text { induction }\end{array}$} & Fentanyl group & Ketamine group & \multirow[b]{2}{*}{$P$ value } \\
\hline & \multicolumn{2}{|c|}{ Mean $( \pm$ SD } & \\
\hline & \multicolumn{2}{|c|}{ SBP (mmHg) } & \\
\hline $1 \mathrm{~min}$ & $99.4(8.5)$ & $106.7(11.3)$ & $<0.001$ \\
\hline $2.5 \mathrm{~min}$ & $96.8(8.2)$ & $107.1(11.3)$ & $<0.001$ \\
\hline $5 \mathrm{~min}$ & $103.0(8.1)$ & $110.7(8.1)$ & $<0.001$ \\
\hline $7.5 \mathrm{~min}$ & $98.6(8.8)$ & $113.7(10.2)$ & $<0.001$ \\
\hline \multirow[t]{2}{*}{$10 \mathrm{~min}$} & $99.0(5.9)$ & $111.9(7.3)$ & $<0.001$ \\
\hline & \multicolumn{2}{|c|}{ DBP (mmHg) } & \\
\hline $1 \mathrm{~min}$ & $59.6(7.1)$ & $67.5(8.8)$ & $<0.001$ \\
\hline $2.5 \mathrm{~min}$ & $59.1(7.0)$ & $67.3(8.9)$ & $<0.001$ \\
\hline $5 \mathrm{~min}$ & $64.2(7.6)$ & $70.0(7.6)$ & $<0.001$ \\
\hline $7.5 \mathrm{~min}$ & $62.3(8.3)$ & $72.0(7.6)$ & $<0.001$ \\
\hline \multirow[t]{2}{*}{$10 \mathrm{~min}$} & $63.5(8.0)$ & $69.6(7.9)$ & $<0.001$ \\
\hline & \multicolumn{2}{|c|}{ MAP (mmHg) } & \\
\hline $1 \mathrm{~min}$ & $72.9(6.9)$ & $80.5(9.4)$ & $<0.001$ \\
\hline $2.5 \mathrm{~min}$ & $71.7(6.8)$ & $80.4(9.6)$ & $<0.001$ \\
\hline $5 \mathrm{~min}$ & $77.1(7.2)$ & $83.6(7.4)$ & $<0.001$ \\
\hline $7.5 \mathrm{~min}$ & $74.4(8.0)$ & $85.8(8.3)$ & $<0.001$ \\
\hline $10 \mathrm{~min}$ & $75.3(6.2)$ & $83.5(6.0)$ & $<0.001$ \\
\hline
\end{tabular}

Mean systolic blood pressures were higher in the Ketamine arm compared to the Fentanyl arm over the duration of follow up; Ketamine $\beta$-coefficient $=7.95$ (5.96, 9.95), $\mathrm{p}<0.001$. On assessing the absolute differences in mean values, this was not seen as clinically significant. The significant outcome measure was the difference from baseline rather the difference between the groups. Similarly linear regression done comparing mean MAPs (controlling for sex, age and ASA status) showed higher mean MAP in the Ketamine group compared to the Fentanyl group during the period of follow up; Ketamine $\beta$-coefficient $=6.63(5.06,8.20), \mathrm{p}<0.001$. Again, on assessing the absolute differences in mean values, this was not seen as clinically significant. The significant outcome measure was the difference from baseline rather the difference between the groups.

Higher mean diastolic blood pressure were seen in the ketamine arm during the period of follow up, Ketamine $\beta$-coefficient $=6.11(4.67,7.55), p<0.001$. Once again, on assessing the absolute differences in mean values, this was not seen as clinically significant. The significant outcome measure was the difference from baseline rather the difference between the groups. 
Even though the hypertensive episodes observed in the Ketamine group did not reach statistical significance, survival probability against hypertension was analysed and it shows faster development of hypertension in those treated with Ketamine, Logrank test, $\mathrm{p}<0.001$. Of note is that in the 10 minute period both the Ketamine and Fentanyl groups had a less than $15 \%$ probability of developing hypertension.

A total of $13(12 \%)$ episodes of tachycardia ( $>20 \%$ increase in baseline heart rate) were seen; $7 / 54(13 \%)$ in the Ketamine group and 6/54(11\%) in the Fentanyl group, showing no significant difference in incidence of tachycardia, $\mathrm{x} 2=0.05, \mathrm{p}=0.82$.

When only considering the means, Ketamine arm had higher mean heart rates during follow up compared to Fentanyl arm despite similar baseline measurement as summarised in table 4. These were however not deemed clinically significant; largest difference between the two groups $=5.4 \mathrm{bpm}$. After adjusting for age, sex and ASA status, use of Ketamine was not significantly associated with tachycardia during follow up, odds ratio=1.14 (95\% confidence interval $0.35,3.67)$. Linear regression done comparing heart rates (controlling for sex, age and ASA status) showed higher mean heart rates in Ketamine group compared to Fentanyl group during the period of follow, Ketamine $\beta$-coefficient $=2.26(0.66,3.86), p=0.006$. This was not considered clinically significant: mean heart rates for both groups ranged between 70 and $80 \mathrm{bpm}$ in both groups.
Overall, 62(57\%) of participants developed hypotension (fall $>20 \%$ post baseline) during follow up. This was more common in those who received Fentanyl, 41/54 (76\%), compared to Ketamine recipients, 21/54 (39\%), $\mathrm{X} 2=16.9, \mathrm{p}<0.001$. This suggests a trend towards more post-induction hypotension with Fentanyl use (the study was not powered to detect this difference). The use of Fentanyl was associated with more episodes of hypotension, adjusted odds ratio $=5.5(95 \%$ confidence interval $2.2,13.9)$ - adjustment made for age, sex and ASA status. Conversely, the use of Ketamine was associated with less episodes of hypotension, adjusted odds ratio $=0.18$ (95\% confidence interval $0.07,0.45$ ).

Hypotension was seen from 1 minute of observation whether measured by systolic, diastolic, or mean blood pressures in both Ketamine and Fentanyl arm and the significantly higher episodes in the Fentanyl arms were seen from 1 minute and persisted throughout follow up.

The Survival probability against hypotension was analysed and it shows faster time to development of hypotension in the Fentanyl arm during period of follow up, Logrank test, $\mathrm{p}<0.001$, with more than $75 \%$ probability of developing hypotension by 10 minutes in the Fentanyl. No rescue interventions were employed within the time of the study and no adverse events were recorded.

There was no report of any patient with hallucinations or confusion deemed to be outside of the realm of post general anaesthesia emergence behaviour. 
Table 4: Comparison of heart rate between fentanyl and ketamine groups

\begin{tabular}{|c|c|c|c|}
\hline \multirow{2}{*}{ Time (min) post-induction } & Fentanyl group & Ketamine group & \multirow[b]{2}{*}{ p value } \\
\hline & \multicolumn{2}{|c|}{$\begin{array}{l}\text { Heart Rate (bpm) } \\
\quad \operatorname{Mean}( \pm \text { SD })\end{array}$} & \\
\hline $1 \mathrm{~min}$ & $74.7(10.1)$ & $75.2(10.7)$ & 0.795 \\
\hline $2.5 \mathrm{~min}$ & $74.9(9.7)$ & $75.5(9.9)$ & 0.765 \\
\hline $5 \mathrm{~min}$ & $75.2(9.9)$ & $79.8(10.3)$ & 0.02 \\
\hline $7.5 \mathrm{~min}$ & $71.6(9.8)$ & $77.0(9.8)$ & 0.005 \\
\hline $10 \mathrm{~min}$ & $70.1(8.9)$ & $74.7(11.3)$ & 0.021 \\
\hline
\end{tabular}

\section{Discussion}

As far as our literature review went, this is the first study investigating the use of the combination of Ketamine and Propofol versus Fentanyl and Propofol, at the cited doses, to attenuate the haemodynamic response to laryngoscopy and endotracheal intubation. The dosing of Propofol and Fentanyl forms part of the standard of care at our hospital. No rescue interventions were employed within the time of the study and no adverse events were recorded.

We postulated that at an appropriate (and as yet unknown) dose, the Ketamine dose would be high enough to confer adequate analgesia needed to reduce the untoward haemodynamic responses to laryngoscopy and endotracheal intubation and low enough to not manifest its own cardio-stimulant effects while preventing the commonly observed drop in blood pressure immediately post-induction (pre-laryngoscopy).

In this study, laryngoscopy and endotracheal intubation were performed 5 minutes after the administration of the induction medication. This was assumed to allow for peak effect of these medications to be attained.
The key finding of this study was that the occurrence of hypertension (defined as an increase of $20 \%$ from baseline values) post laryngoscopy and endotracheal intubation was not significantly higher, $\mathrm{p}=0.06$ (statistically or in the author's opinion, clinically) with the use of $0.5 \mathrm{mg} / \mathrm{kg}$ Ketamine than with $1.0 \mu \mathrm{g} / \mathrm{kg}$ Fentanyl use. For instance, there was an episode of increase in SBP of $100 \mathrm{vs} 121 \mathrm{mmHg}$ at 7.5 minutes, of MAP from 96 to $118 \mathrm{mmHg}$ at 7.5 minutes. On controlling for differences in age, sex and ASA status, Ketamine still did not show an association with hypertension. Mean blood pressures were higher in the Ketamine than the Fentanyl group but this was not deemed clinically significant, the greatest difference being a range of 88.6 to $105 \mathrm{mmHg}$ versus a range of 95.8 to $118.4 \mathrm{mmHg}$ in SBP at 2.5 minutes; 66.4 to $82.4 \mathrm{mmHg}$ versus 77.5 to $93.6 \mathrm{mmHg}$ in $\mathrm{MAP}$ at 7.5 minutes; 52.1 to $66.1 \mathrm{mmHg}$ versus 58.4 to $76.2 \mathrm{mmHg}$ in DBP at 2.5 minutes for Fentanyl and Ketamine groups respectively.

On analysis, there was no statistically significant increase in heart rate from baseline with use of either drug combination, $\mathrm{p}=0.82$. On controlling for differences in age, sex and ASA status, Ketamine still did not show an as- 
sociation with tachycardia. The study was however not powered to detect differences in the secondary outcome measures. The mean heart rates in the Ketamine group were higher than in the Fentanyl group, but, this was not deemed clinically significant; the greatest difference in range of mean heart rate was 61.8 to $81.4 \mathrm{bpm}$ versus 67.2 to $86.8 \mathrm{bpm}$ in the Fentanyl and Ketamine group respectively at 7.5 minutes post induction.

Post induction hypotension also lends to the risk of myocardial ischaemia and is often seen with opioid based anaesthesia induction. There was a statistically significant decrease in blood pressure (occurrence of hypotension) post induction (pre-laryngoscopy) in the Fentanyl group compared to the Ketamine group, $\mathrm{p}=<0.001$. Again, this study was not powered to detect differences in this secondary outcome measure. On adjusting for confounders, Ketamine was still associated with less episodes of post induction hypotension. The post induction hypotension observed is likely secondary to peripheral vasodilatory effect of Propofol as well as the direct cardio-depressant effects of Fentanyl.

It is worth noting that the participants' preoperative fluid status was unknown and as such, some of them may have been relatively fluid depleted. This may have unmasked a relative intravascular hypovolemia in these patients and exaggerated the association of either drug with this observation.

After laryngoscopy and endotracheal intubation, Hayakama-Fujii et al demonstrated a significantly greater increase in blood pressure and heart rate above baseline values in the Propofol-Ketamine group. Patients received Propofol at $2 \mathrm{mg} / \mathrm{kg}$ plus either Fentanyl $3.0 \mu \mathrm{g} / \mathrm{kg}$, Ket-

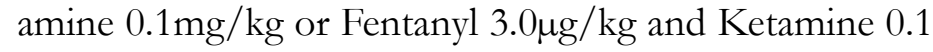
$\mathrm{mg} / \mathrm{kg}^{18}$. We postulate that the reason for this difference from our findings is that the doses are not equianalgesic (the Ketamine dose is too low to allow for its analgesia to be effective versus the higher dose of Fentanyl used). Similar to our study, Singh at al demonstrated statistically significant increases in mean systolic and diastolic blood pressures at all times until 10 minutes post-induction with a Propofol-Ketamine combination than with a Propofol-Fentanyl combination ${ }^{4}$. That study did not however report analysis of variation of these haemodynamic parameters from baseline. This may not have been significant, even if the difference in the means was. Furthermore, it is worth commenting on whether the differences observed are of clinical significance (their study demonstrated $\leq 14 \mathrm{mmHg}$ difference in SBP 122.16 versus 132.68 and $\leq 11 \mathrm{mmHg}$ difference in DBP 75.20 versus 86.92 for Fentanyl and Ketamine groups respectively within a comparable study period time).

Like our study, the same study demonstrated a statistically significant increase in pulse rate post induction with Propofol-Ketamine than with Propofol-Fentanyl. Again, this was a difference in mean heart rates rather than from baseline heart rate. The greatest difference in heart rate was $14 \mathrm{bpm}$ (90 bpm versus $76 \mathrm{bpm}$ in the Ketamine versus Fentanyl groups respectively at 10 minutes post induction). They also demonstrated a statistically significant fall in SBP and DBP with the Fentanyl group in the post-induction pre-laryngoscopy period.

In their study however, Propofol was used at a dose of $1.0 \mathrm{mg} / \mathrm{kg}$ when combined with Ketamine at a dose of $1.0 \mathrm{mg} / \mathrm{kg}$ against Propofol $1.5 \mathrm{mg} / \mathrm{kg}$ versus Fentanyl at $2.0 \mu \mathrm{g} / \mathrm{kg}$. The anaesthetic dose of Ketamine is quoted as being between 1.0 and $2.0 \mathrm{mg} / \mathrm{kg}$ and as such it could be postulated that the relatively high dose of ketamine allowed for its sympathomimetic effects to manifest. In addition, the Propofol dose was on the lower range of anaesthetic dosing (usually $1.0-2.0 \mathrm{mg} / \mathrm{kg}$ ) and may also have been too low to consistently provide adequate depth of anaesthesia.

Goyal et al compared the two drugs at similar (but not equal) dosages for short surgical procedures ${ }^{19}$. One group was given Ketamine $0.5 \mathrm{mg} / \mathrm{kg}$ and the other Fentanyl $1.5 \mu \mathrm{g} / \mathrm{kg}$ and 2 minutes later given Propofol $2.5 \mathrm{mg} /$ $\mathrm{kg}$. Laryngeal mask airways were used in this study but post-induction, pre-intubation haemodynamic variables were noted. There was significant decrease $(\mathrm{P}<0.05)$ in the systolic and diastolic blood pressure at 1, 3, and 5 minutes in the Fentanyl group whereas the change was insignificant at 10 minutes. This corresponds with the results of our study. However, it should be noted that at 1 minute, Propofol had not been administered and at 3 minutes the laryngeal mask airway was likely already being inserted. The difference in methodology renders it difficult to compare those results with the results of this study. 
In a study by Honarmand et al, the largest difference between the Thiopental-Fentanyl and Thiopental-Ketamine groups in any blood pressure parameter was $6.7 \mathrm{mmHg}$ (109.2 versus $115.09 \mathrm{SBP}$ ) and a heart rate difference of 16.2bpm (69.7 versus 85.9$)$, which was statistically significant (17). In that study, Thiopental was used at $4.0 \mathrm{mg} /$ $\mathrm{kg}$, Fentanyl at $3.0 \mu \mathrm{g} / \mathrm{kg}$ and Ketamine at $0.1 \mathrm{mg} / \mathrm{kg}$. Again, this represented a difference in means and not a difference from baseline values. Nevertheless, any difference in variation from baseline that may exists between the two studies may be attributed to the sub-optimal of ketamine versus a supra-optimal of Fentanyl which may have consequently produced lower analgesic effects and thus less likely to attenuate the pressor response to laryngoscopy and endotracheal intubation.

The observations made in our study may, like our assumption while choosing the Ketamine protocol dose, be attributed to Ketamine being at a low enough dose that its sympathomimetic effects are minimal and still high enough to confer effective analgesia deemed necessary to attenuate the response to laryngoscopy and endotracheal intubation. Propofol was used at a dose of $2.0 \mathrm{mg} / \mathrm{kg}$ to increase the likelihood of adequate depth of anaesthesia. The findings in this study are in keeping with our null hypothesis: there is no difference in the occurrence of hypertension post laryngoscopy and endotracheal intubation with the use of Ketamine versus Fentanyl.

According to the study protocol, various rescue actions and medications were to be employed in the case of persistent hypertension or tachycardia. This was not observed in any of the groups.

There was no report of any patient with hallucinations or confusion deemed outside of the realm of post general anaesthesia behavior. This data was however not acquired by use of a validated tool. Also, midazolam was sometimes administered within the course of the anaesthetic (but only after the 10 minutes post induction and before emergence). As such, it is possible that the $0 \%$ occurrence of these neuropsychiatric manifestations may have been suppressed by benzodiazepine use. Literature does however show that such adverse effects are minimal to nil with use of low dose ketamine and with the combined use with Propofol.

\section{Strengths}

After a thorough literature review, it appears that following years of research into methods to attenuate the hae- modynamic response to laryngoscopy and endotracheal intubation, this is the first prospective, double blinded randomized control trial to employ the study drugs at these dose combinations using this methodology. It is thus hoped that these findings will add to the fund of knowledge concerning maintaining cardiovascular and haemodynamic stability in the peri-intubation period of the general anaesthetic.

\section{Limitations}

This was not a multi-centre trial. This may affect the generalizability of the results obtained from this study. The study was powered based on a small number of single centre studies that had similar sample sizes. It was not powered to analyse the effect of the study drugs on tachycardia and post-induction hypotension.

Depth of anaesthesia may have varied as neither the use of bispectral index (BIS) nor electroencephalogram (EEG) was employed. As such, the susceptibility to the adverse haemodynamic responses to laryngoscopy and endotracheal may have been higher in some patients due to inadequate depth of anaesthesia.

By general Anaesthesiology protocol, patients are required to fast for at least 6 hours prior to induction of anaesthesia. Some participants in this study may have fasted for a longer time. Use of Propofol (known to cause peripheral vasodilatation) could unmask a relative intravascular hypovolemia. The patients' preoperative haemodynamic status was thus unknown and may have contributed to the occurrence of post induction hypotension.

The logistical and budgetary constraints of this study would not allow for 12 lead electrocardiography monitoring during the study period. The inclusion of this monitoring would aide in identifying episodes of myocardial ischaemia, which is what the continued search for an "ideal" induction drug or drug combination attempts to address.

\section{Conclusion}

Based on the results of this study, we conclude that there is no difference in the occurrence of hypertension and tachycardia with the use of Ketamine at $0.5 \mathrm{mg} / \mathrm{kg}$ in combination with Propofol at $2.0 \mathrm{mg} / \mathrm{kg}$. In this regard, Ketamine provides a viable alternative to Fentanyl at 1.0 $\mu \mathrm{g} / \mathrm{kg}$ for attenuating the pressor response to laryngoscopy and endotracheal intubation. Additionally, our results suggest that Ketamine may protect against post-induction hypotension. 


\section{Conflict of interest}

None declared.

\section{References}

1. Pouraghaei M, Moharamzadeh P, Soleimanpour H, Rahmani $\mathrm{F}$ et al. Comparison between the effects of alfentanil, fentanyl and sufentanil on hemodynamic indices during rapid sequence intubation in the emergency department. Anesth Pain Medicine. 2014 Jan 25; 4(1): e14618 2. Reich DL, Hossa S, Krol M, Baez B, Patel P, et al . Predictors of hypotension after induction of general anaesthesia. Anesth Analg. 2005 Sep; 101(3):622-8

3. Feng C, Qi SH, Zou YM, Ma XS, Gao DP, Han BQ. Effects of dexmedetomidine combined with fentanyl in patients undergoing anesthesia induction by sevoflurane. Zhonghua Yi Xue Za Zhi. 2012 Jul 17; 92(27):1889-91

4. Singh Bajwa SJ, Bajwa SK, Kaur J. Comparison of two drug combinations in total intravenous anesthesia: Propofol-ketamine and propofol-fentanyl. Saudi J of Anaesth. 2010; May ;4(2): 72-79

5. Gupta K, Girdhar KK, Anand R, Majgi SM, et al. Comparison Of Haemodynamic Responses To Intubation: Flexible Fibreoptic Bronchoscope Versus Bonfils Rigid Intubation Endoscope. Indian J Anaesth, 2012 Jul ;56, (4): 353-358

6. Aghdaii N, Azarfarin R, Yazdanian F, Faritus SZ. Cardiovascular responses to orotracheal intubation in patients undergoing coronary artery bypass grafting surgery comparing fiberoptic bronchoscopy with direct laryngoscopy. Middle East J Anaesthesiol. 2010 Oct; 20(6):833-8.

7. Arora S, Kulkarni A, Bhargava AK. Attenuation of hemodynamic response to laryngoscopy and orotracheal intubation using intravenous clonidine. J Anaesthesiol Clin Pharmacol. 2015 Jan-Mar; 31(1): 110-114

8. Charuluxananan S, Kyokong O, Somboonviboon W, Balmongkon B, Chaisomboonpan S. Nicardipine versus lidocaine for attenuating the cardiovascular response to endotracehl intubation. J Anesth. 200 Apr 25; 14(2):77-81 9. Gunes Y, Gündüz M; Özcengiz D; Özbek H; Isik G Dexmedetomidine-remifentanil or propofol-reminfentanil anesthesia in patients undergoing intracranial surgery. Neurosurg Q. 2005; 15(2):122-6

10. Menda F, Koner O, Sayin M, Ture H et al Dexmedetomidine as an adjunct to anesthetic induction to attenu- ate hemodynamic response to endotracheal intubation in patients undergoing fast-track CABG. Ann Card Anaesth. 2010 Jan-Apr, 13(1):16-21

11. Powroznyk AV, Vuylsteke A, Naughton C, Misso SL, Holloway J et al Comparison of clevidipine with sodium nitroprusside in the control of blood pressure after coronary artery surgery. Eur J Anaesth. 2003 Sep;20(9):697703

12. Abou-Arab MH, Heier T. Caldwell JE. Dose of alfentanil needed to obtain optimal intubation conditions during rapid-sequence induction of anaesthesia with thiopentone and rocuronium. Br J Anaesth. 2007 May; 98(5):604-10

13. Hall JE, Uhrich TD, Ebert TJ. Sedative, analgesic and cognitive effects of clonidine infusions in humans. $\mathrm{Br} J$ Anaesth. 2001 Jan; 86(1):5-11

14. Shailaja S, Srikantu J. Comparison of effect of esmolol vs esmolol and fentanyl on hemodynamic response to laryngoscopy and tracheal intubation in controlled hypertensive patients: a randomized controlled double blind study. Anaesthesia, Pain and Intensive Care. 17(3):267-273

15. Satsumae T, Yamaguchi H, Sakaguchi M, Yasunaga T, Yamashita $\mathrm{S}$ et al . Preoperative small-dose ketamine prevented tourniquet-induced arterial pressure increase in orthopedic patients under general anesthesia. Anesth Analg. 2001 May ; 92(5):1286-9

16. Khan FA, Ullah H. Pharmacological agents for preventing morbidity associated with the haemodynamic response to tracheal intubation. Cochrane Database Syst Rev. 2013 Jul 3;(7):CD004087

17. Honarmand A, Safavi M, Cardiovascular Response during Induction of Anesthesia and Tracheal Intubation with Thiopental added to Fentanyl, Ketamine, and Fentanyl-Ketamine. Ghana Med J. 2009 Mar; 43(1): 7-12

18. Hayakawa-Fujii Y, Takada M, Ohta S, Dohi S. Hemodynamic stability during induction of anesthesia and tracheal intubation with propofol plus fentanyl, ketamine, and fentanyl-ketamine. J Anesth. 2001; 15(4):191-6

19. Goyal R, Singh M, Sharma J. Comparison of ketamine with fentanyl as co-induction in propofol anesthesia for short surgical procedures. Int J Crit Illn Inj Sci. 2012 Jan; 2(1):17-2

20. Kurdi MS, Theerth KA, Deva RS. Ketamine: current applications in anesthesia, pain, and critical care. Anesth Essays Res. 2014 Sep-Dec; 8(3): 283-290 